\title{
Aberrant methylation of RASGRF2 and RASSF $1 A$ in human non-small cell lung cancer
}

\author{
HONG CHEN $^{1,3}$, MAKOTO SUZUKI ${ }^{1,4}$, YOHKO NAKAMURA ${ }^{2}$, MIKI OHIRA ${ }^{2}$, \\ SOICHIRO ANDO ${ }^{1}$, TOMOHIKO IIDA ${ }^{1}$, TAKAHIRO NAKAJIMA ${ }^{1}$, \\ AKIRA NAKAGAWARA $^{2}$ and HIDEKI KIMURA ${ }^{1}$
}

\begin{abstract}
Divisions of ${ }^{1}$ Thoracic Diseases and ${ }^{2}$ Biochemistry, Chiba Cancer Center, 666-2 Nitona, Chuoh-ku, Chiba $260-8717 ;{ }^{3}$ Department of Pulmonary Disease, The First Affiliated Hospital, Chongqing University of Medical Sciences, 1 Youyi Road, Yuzhong District, Chongqing, 400016, P.R. China
\end{abstract}

Received February 9, 2005; Accepted April 16, 2005

\begin{abstract}
Aberrant methylation of promoter $\mathrm{CpG}$ that causes silencing of tumor suppressor genes (TSGs) may play a key role in the carcinogenesis of many cancer types. RASSF1A, regarded as a TSG, has been extensively studied in lung cancer and other malignant tumors, whereas RASGRF2 has only been reported to possibly play a role in the pathogenesis of pancreatic cancer cell lines. The aims of our study were to i) determine the methylation profile of $R A S G R F 2$ and ii) compare the methylation profiles of RASGRF2 with RASSF1A in lung cancer. We examined RASGRF2 expression by reverse transcription PCR and aberrant methylation of $R A S G R F 2$ by methylation-specific PCR in lung cancer cell lines. Loss of RASGRF2 expression was presented in $36 \%$ lung cancer cell lines while aberrant methylation of RASGRF2 was present in 30\% (3/10) non-small cell lung cancer (NSCLC) cell lines and in 25\% (1/4) small cell lung cancer (SCLC) cell lines. The concordance between loss of expression and aberrant methylation of RASGRF2 was $86 \%$ (12/14). RASGRF2 expression was restored after treatment with the demethylating agent, 5-aza-2'-deoxycytidine in all four cell lines tested that downregulated RASGRF2 expression. Among primary NSCLC, RASGRF 2 and RASSF 1A methylation was observed in 34\% (39/114) and $39 \%(44 / 114)$ of cases respectively, while it was observed in only $7 \%$ (4/57) and none of the corresponding non-malignant lung tissue. There is no correlation between RASGRF2 and RASSF 1A methyl-ation status. Both RASGRF2 and RASSF1A methylation did not associate with clinical characteristics. Frequent methylation and silencing of RASGRF2 in tumor
\end{abstract}

Correspondence to: Dr Makoto Suzuki, ${ }^{4}$ Present address: Department of Thoracic Surgery, Graduate School of Medicine, Chiba University, 1-8-1 Inohana, Chuoh-ku, Chiba 260-8677, Japan

E-mail: makotosuzuki@hotmail.co.jp

Key words: RASGRF2, RASSF1A, methylation, lung cancer cells may play an important role, different from that of RASSF 1A, in the carcinogenesis of NSCLC.

\section{Introduction}

Aberrant methylation of promoter $\mathrm{CpG}$ that causes silencing of tumor suppressor genes (TSGs) may play a key role in the carcinogenesis of many cancer types. Recent advances in molecular genetics have showed that multiple TSGs are involved in human lung cancer pathogenesis (1).

Ras association domain family $1 \mathrm{~A}(\mathrm{RASSF} 1 \mathrm{~A})$ is located within a $120-\mathrm{kb}$ region of chromosome $3 \mathrm{p} 21$, a region that is epigenetically inactivated at high frequency in non-small cell lung cancer (NSCLC) (2). Further studies reveal that, in addition to loss of heterozygosity ( $\mathrm{LOH}$ ), promoter hypermethylation of RASSF1A is another mechanism of this gene's loss of expression (3-5). It has been shown that RASSF 1A bind to the Ras-GTP binding protein, Nore1, which is consistent with the role of a negative effector of Ras oncoprotein (6). RASSF1A could induce cell cycle arrest by engaging the RB cell cycle checkpoint. Inhibition of cyclin D1 by RASSF 1A occurs post-transcriptionally and is likely at the level of translational control. These results strongly suggest that RASSF $1 A$ is an important human tumor suppressor protein acting at the level of G(1)/S-phase cell cycle progression (7).

Ras protein-specific guanine nucleotide-releasing factor 2 (RASGRF2) was originally found by Anborgh et al (8); who, using a yeast 2-hybrid screen of a brain cDNA library with the Dbl homology (DH) domain of RASGRF1 as bait, followed by 5-prime and 3-prime RACE, isolated a full-length cDNA encoding RASGRF2. RASGRF2 is involved in H-Ras signaling (9) and abundantly expressed in a variety of tissue, such as brain, heart and lung (8). However, its expression is decreased in rat mammary carcinomas (10). Recently, it was reported that $R A S G R F 2$ was abundantly expressed in at least one of two ductal epithelial cell lines, but there was loss of expression due to promoter methylation in pancreatic cancer cell lines, using high-throughput microarray analysis (11). Among the 12 genes silenced by methylation of 5 ' regions, RASGRF2 was methylated at about $17 \%$, which is higher than a few other genes. This gene is located at a region of chromosome 
Table I. Clinical characteristics and RASGRF2 and RASSF1A methylation of lung cancer patients.

\begin{tabular}{|c|c|c|c|c|c|}
\hline Clinical factors & No. of cases & $\begin{array}{c}\text { No. of } R A S G R F 2 \\
\text { methylation }(\%)\end{array}$ & p-value ${ }^{a}$ & $\begin{array}{l}\text { No. of RASSF } 1 A \\
\text { methylation }(\%)\end{array}$ & p-value ${ }^{a}$ \\
\hline \multicolumn{6}{|l|}{ Gender } \\
\hline Male & 65 & $25(38)$ & 0.32 & $24(37)$ & 0.70 \\
\hline Female & 49 & $14(29)$ & & $20(41)$ & \\
\hline \multicolumn{6}{|l|}{ Age } \\
\hline$\leq 66^{\mathrm{b}}$ & 58 & $20(34)$ & $>0.99$ & $19(33)$ & 0.25 \\
\hline$>66$ & 56 & $19(34)$ & & $25(45)$ & \\
\hline \multicolumn{6}{|l|}{ Smoke } \\
\hline Never & 42 & $12(29)$ & 0.41 & $18(43)$ & 0.55 \\
\hline Smoker & 72 & $27(38)$ & & $26(36)$ & \\
\hline \multicolumn{6}{|l|}{ Histology } \\
\hline Adenocarcinoma & 85 & $28(38)$ & & $36(42)$ & \\
\hline Squamous cell carcinoma & 27 & $11(41)$ & $0.30^{c}$ & $7(26)$ & 0.10 \\
\hline Others (ad-sq, large) & 2 & 0 & & $1(50)$ & \\
\hline \multicolumn{6}{|l|}{ PT } \\
\hline $\mathrm{T} 1$ & 50 & $15(30)$ & 0.26 & $19(38)$ & 0.53 \\
\hline $\mathrm{T} 2,3,4$ & 64 & $24(38)$ & & $25(39)$ & \\
\hline \multicolumn{6}{|l|}{$\mathrm{pN}$} \\
\hline No & 68 & $25(37)$ & 0.31 & $24(35)$ & 0.86 \\
\hline $\mathrm{N} 1,2,3$ & 46 & $14(30)$ & & $20(43)$ & \\
\hline \multicolumn{6}{|l|}{ pStage } \\
\hline $\mathrm{I}$ & 50 & $18(36)$ & 0.71 & $19(38)$ & 0.53 \\
\hline II, III, IV & 64 & $21(33)$ & & 25 (39) & \\
\hline
\end{tabular}

${ }^{a}$ Fisher's exact probability test; ${ }^{b}$ Divided into two groups by median age; ${ }^{c}$ Adenocarcinoma vs. squamous cell carcinoma; Ad-sq, adenosquamous cell carcinoma.

$5 q 13$, a locus which frequently shows an allelic imbalance in lung cancer, and was speculated to act as a TSG (12). This prompted us to examine the methylation status of RASGRF2 in lung cancer.

Imbalance of the Ras signaling pathway is a major hallmark of human cancer. Activated Ras proteins interact with a broad range of effector proteins to induce a diverse series of biological consequences. RASSF $1 A$ and RASGRF2, correlating with Ras-effector, were investigated in this study. We examined the methylation, by methylation-specific PCR (MSP), of RASSF1A and RASGRF2 in lung cancer cell lines, and analyzed the methylation status of primary lung cancer, and correlated them with the clinicopathological features. We examined the mRNA expression of RASGRF2 by reverse transcription PCR (RT-PCR) and treated those cell lines which showed loss of RASGRF2 mRNA expression with 5-Aza-CdR.

\section{Materials and methods}

Cell lines. Ten non-small cell lung cancer (NSCLC) and 4 small cell lung cancer (SCLC) cell lines were used in this study. These cell lines were established and provided by Dr Adi F. Gazdar. Cell lines were grown in RPMI-1640 medium supplemented with $5 \%$ fetal bovine serum and incubated in $5 \% \mathrm{CO}_{2}$ at $37^{\circ} \mathrm{C}$. Normal tracheal RNA was obtained from Clontech (Palo Alto, CA), and non-malignant human bronchial epithelial cells (NHBEC) were cultured as reported previously (unpublished data).

Clinical samples. Surgically resected specimens of 114 patients with primary lung cancer and 57 adjacent non-malignant lung tissue samples were obtained from Chiba Cancer Center, Japan, after obtaining Institutional Review Board approval and informed consent. Samples were immediately frozen and stored at $-80^{\circ} \mathrm{C}$ until used. The clinical characteristics of these patients are detailed in Table I.

Methylation assay. Genomic DNA was obtained from lung cancer cell lines, cultured non-malignant cells, primary tumors and adjacent non-malignant tissue, by digestion with $20 \mathrm{mg} / \mathrm{ml}$ proteinase K (Life Technologies, Inc.), followed by standard phenol/chloroform (1:1) extraction and ethanol precipitation (13). One $\mu \mathrm{g}$ of genomic DNA was further subjected to bisulfite treatment following the protocol of EZ DNA methylation kit (ZYMO Research, Orange, CA). The modified DNA was used as a template for MSP. DNA methylation patterns in the $\mathrm{CpG}$ island of RASGRF2 were determined by the method of MSP as reported previously (11). Primer sequences of RASGRF2 for the unmethylated reaction were: 5'-TGGAGAGTGTGTTTTGGTTTTA-3' (sense), and 5'-CCAACCAACAAAAAACACCCC-3' (antisense), which amplify a 117 -bp product. Primer sequences of 
RASGRF2 for the methylated reaction were: 5'-GGCGGAGAG CGTGTTTC-3' (sense), and 5'-CCGACCGACGAAAAACG-3' (antisense), which amplify a 119-bp product. Primer sequences of RASSF 1A for the unmethylated reaction were: 5'-GGTT TTGTGAGAGTGTGTTTAG-3' (sense), and 5'-CACTAACA AACACAAACCAAAC-3' (antisense). Primer sequences of RASSF $1 A$ for the methylated reaction were: 5'-GGGTTTTGCG AGAGCGCG-3' (sense), and 5'-GCTAACAAACGCGAA CCG-3' (antisense). Universal methylated DNA (Chemicon, CA), which was subjected to bisulfite treatment, was used as a positive control for methylated alleles. Negative controls without DNA were included in each assay. Nine $\mu 1$ of each PCR product was loaded on $2 \%$ agarose gels stained with ethidium bromide. Results were confirmed by repeating bisulfite treatment and MSP for all samples.

\section{Reverse transcription-PCR for gene expression}

$R A S G R F 2$. mRNA expression was analyzed by RT-PCR. Total RNA was obtained from cell lines (NHBEC, 10 NSCLC and 4 SCLC), primary tumors and adjacent non-malignant tissue by single-step method. Reverse transcription reaction was performed on $5 \mu \mathrm{g}$ of total RNA with the SuperScriptII First-Strand Synthesis using oligo (dT) primer system (Life Technologies Inc.), and aliquots of the reaction mixture were used for the subsequent PCR amplification. Expression of B-actin was used as an internal control to confirm the success of the reverse transcription reaction. The forward PCR amplification primer of RASGRF2 was 5'-ACATTTTGATTGA GAGGGAAGT-3', and the reverse primer 5'-CGCATTCGTT CTTTGGTTT-3'. The forward PCR amplification primer of B-actin was 5'-CAACTGGGACGACATGGAGA-3', and the reverse primer 5'-ACGTACATGGTGGGGTGTTG-3'. These primer sequences were identical to the human target genes, as was confirmed by BLAST searches. PCR products were analyzed on $2 \%$ agarose gels stained with ethidium bromide. NHBEC and normal trachea were used as normal controls for RT-PCR.

5-aza-2'-deoxycytidine (5-Aza-CdR) treatment. Methylated cell lines that downregulated gene expression were incubated in culture medium with $1 \mu \mathrm{M}$ of the demethylating agent 5-AzaCdR (Sigma-Aldrich, St. Louis, MO) for 6 days, with medium changes on days 1, 3 and 5. Cells were harvested and RNA was extracted on day 6 .

Statistical analysis. The differences of methylation between two groups were analyzed by using Fisher's exact test. Survival was calculated from the date of initial diagnosis until death or the date of the last follow-up. Survival was analyzed according to the Kaplan-Meier method, and differences in their distribution were evaluated by means of the log-rank test. All $\mathrm{p}$-values are two-sided. The probability value of $\mathrm{p}<0.05$ was regarded as statistically significant.

\section{Results}

Aberrant methylation of RASSF $1 A$ and RASGRF2 in cell lines. Detailed results of aberrant methylation of RASSF1A and RASGRF2 in cell lines are shown in Fig. 1. Aberrant methylation of RASSF $1 A$ was observed in $79 \%$ (11/14) of
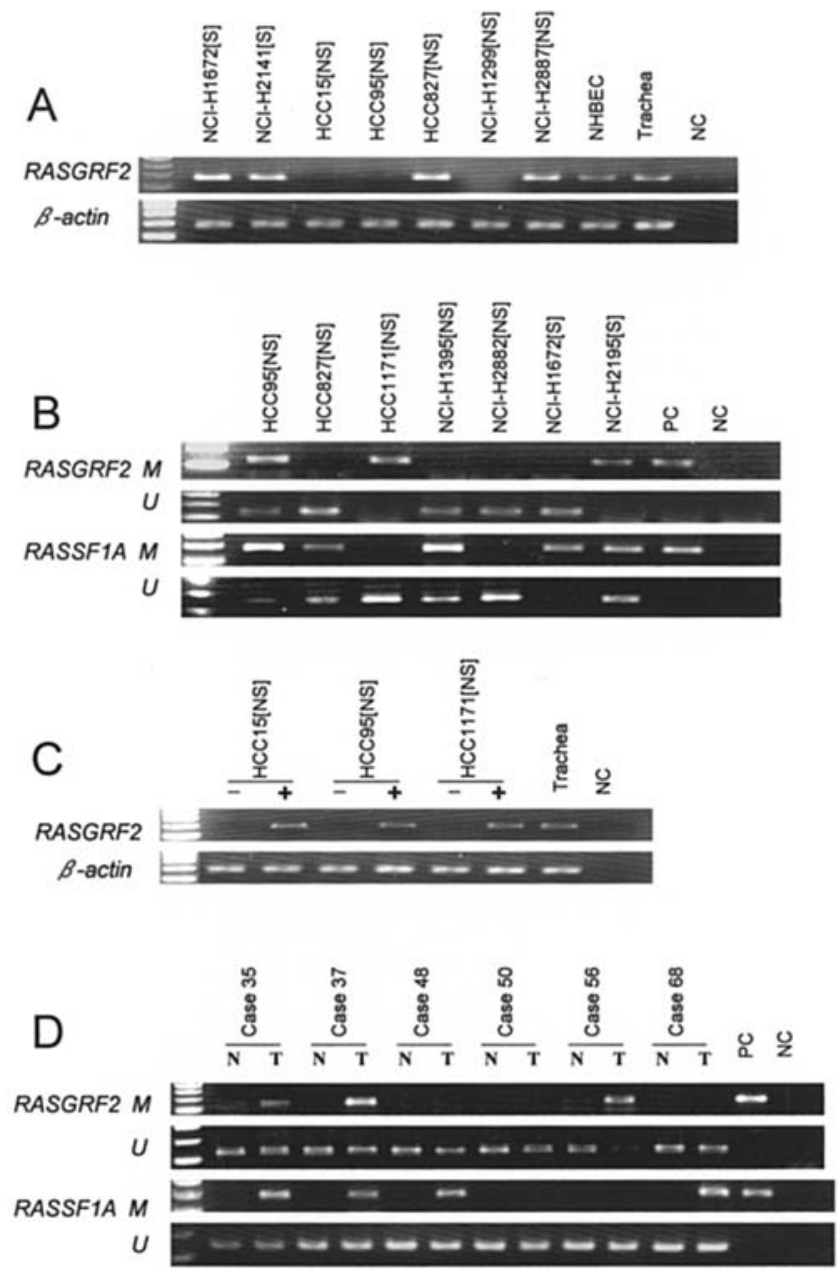

Figure 1. A, Representative examples of RT-PCR assay for RASGRF2 RNA expression in NSCLC and SCLC cell lines. Expression of $\beta$-actin was used as a control for cDNA normalization. NHBEC and trachea were used as positive control. NC, negative control; NS, NSCLC; S, SCLC. Lanes that do not show a band represent samples with loss of expression. B, Methylation analysis of RASGRF2 and RASSF1A in cell lines. Lane M, amplified product with primers recognizing methylated sequence. Lane $\mathrm{U}$, amplified product with primers recognizing unmethylated sequence. $\mathrm{PC}$, positive control; $\mathrm{NC}$, negative control. C, Re-expression of RASGRF2 after treatment with 5-Aza2'-deoxycytidine (5-Aza-CdR). The expression of RASGRF2 lost in those cell lines can be restored after treatment with 5-Aza-CdR. -, Cell line without 5-Aza-CdR; +, Cell line with 5-Aza-CdR; Trachea was used as positive control. D, Representative examples of methylation analysis of RASGRF2 and RASSF $1 A$ in tumor specimens. N, non-malignant lung tissue; $\mathrm{T}$, tumor.

lung cancer cell lines; in 100\% (4/4) of SCLC cell lines and in $70 \%(7 / 10)$ of NSCLC cell lines. Aberrant methylation of both genes was absent in NHBEC. However, aberrant methylation of RASGRF2 was observed in $29 \%$ (4/14) of lung cancer cell lines; in 30\% (3/10) of NSCLC cell lines and $25 \%$ (1/4) of SCLC cell lines. Only two cell lines (NCI-H524 and NCI-H1299) demonstrated loss of expression and lack of methylation of RASGRF2. The concordance between gene expression and methylation of RASGRF2 was $90 \%(9 / 10)$ in NSCLC cell lines, and 75\% (3/4) in SCLC cell lines (overall concordance was $86 \%$ ). There was no correlation between RASGRF2 and RASSF1A methylation status.

Expression of RASGRF2 in cell lines. RASGRF2 expression was examined by RT-PCR, representative examples are shown 
in Fig. 1. Expression of RASGRF2 was present in NHBEC and normal trachea. However, loss of RASGRF2 expression was observed in $36 \%(5 / 14)$ of lung cancer cell lines; in $40 \%$ $(4 / 10)$ of NSCLC cell lines, and $25 \%$ (1/4) of SCLC cell lines.

5-Aza-CdR treatment. To confirm that the promoter methylation was responsible for silencing the RASGRF2 expression, we treated methylated NSCLC cell lines (HCC15, HCC95, HCC1171, NCI-H2195) that showed downregulated RASGRF2 expression with the demethylating agent 5-Aza-CdR. $R A S G R F 2$ expression was restored after the treatment in all four cell lines tested (Fig. 1).

Aberrant methylation of RASSF1A and RASGRF2 in clinical samples. RASSF $1 A$ and RASGRF2 methylation of primary tumors and corresponding non-malignant tissue is detailed in Table I and representative samples are illustrated in Fig. 1. RASSF 1A methylation was observed in 39\% (44/114) of tumors compared to none in corresponding non-malignant tissue. RASGRF2 methylation was observed in $34 \%$ (39/114) of tumors while only $7 \%$ (4/57) was observed in corresponding non-malignant tissue. Methylation of both genes was tumor specific when compared with that of corresponding nonmalignant lung tissue $(\mathrm{p}<0.0001)$. There was no relationship between RASSF $1 A$ methylation and RASGRF2 methylation. RASSF $1 A$ and RASGRF2 methylation with clinicopathological features was also examined. There were no significant correlations with gender, age, smoking history (ever vs. never smoker), or histology (adenocarcinoma vs. squamous cell carcinoma), in either gene. RASSF $1 A$ and $R A S G R F 2$ methylation statuses did not correlate with survival.

Expression of RASGRF2 in clinical samples. We checked 17 clinical samples of RASGRF2 expression by RT-PCR to confirm the relationship between methylation and expression, representative examples are shown in Fig. 1. Expression of $R A S G R F 2$ was present in non-malignant tissue. However, loss of RASGRF2 expression was observed in 53\% (9/17) of NSCLC tumors. The loss of expression was highly correlated with methylation. The concordance between gene expression and methylation of RASGRF2 was $65 \%(11 / 17)$.

\section{Discussion}

Recently, Hagihara et al reported that RASGRF2 is frequently methylated in pancreatic cancer and thought to be a candidate for a TSG (11). However, there is no report about the role of $R A S G R F 2$ in lung cancer. To understand the role of the $R A S G R F 2$ gene in lung cancer, we examined the expression of RASGRF2. RASGRF2 was expressed in tracheal cells, and cultured airway epithelial cells, whereas lung cancer cell lines had $36 \%$ loss of expression. 5-Aza-CdR treatment restored the expression of the gene in RT-PCR-negative cell lines, indicating methylation as a mechanism of transcriptional silencing of the gene. Moreover, methylation of RASGRF2 was present in $34 \%$ of NSCLC with a tumor-specific manner. There are several reports about the correlation between aberrant methylation of TSGs and carcinogenesis of lung cancer (14). RASGRF2 may be added to the list of TSGs. To our knowledge, this is the first report that demonstrates the methylation of RASGRF2 promoter in lung cancer.

We found that RASGRF2 expression was decreased both in lung cancer cell lines and clinical samples, which was the same as in rat mammary carcinomas (10) and in pancreatic cancer cell lines (11). RASGRF2 has been reported to be involved in H-Ras signaling (9). RAS GTPases cycle between an inactive GDP-bound state and an active GTPbound state. As guanine-nucleotide exchange factors, RASGRF2 stimulate the conversion of the GDP-bound form into the active form. However, the methylation changes in our experiment of RASGRF2 were contrary to the changes expected if the H-Ras pathway were activated. RASGRF2 expression has been shown to be nuclear in lung cancer, but little is known about the role of RASGRF2 expression during carcinogenesis. Ras GAP protein generates potent antiapoptotic signals after cleavage by caspase and may play an anti-apoptotic role in tumor cell lines $(15,16)$. Whether $R A S G R F 2$ acts as a TSG by activating apoptotic signals, as well as activating the K-ras gene, is unknown and requires further study.

Inactivation of TSGs may occur via multiple mechanisms, including allelic loss, gene mutation, or by methylation of $\mathrm{CpG}$ sites in promoter regions. RASSFIA is frequently silenced in lung cancer due to promoter hypermethylation in a few studies, while the mutations of RASSF $1 A$ are rare. The frequency of RASSF 1A promoter hypermethylation is about $70-100 \%$ in SCLC tumors whereas it is $30-60 \%$ in NSCLC samples (14). We found hypermethylation of RASSFIA in $100 \%$ (4/4) of SCLC and 70\% (7/10) of NSCLC cell lines, in 39\% (44/114) of primary tumors and none in corresponding non-malignant tissue. The results were highly consistent with the previous reports (14). We did not examine the expression of RASSF 1A because it was confirmed by the previous study (17). However, there was no correlation between RASSF $1 A$ and RASGRF2 methylation status. The reason might be that RASSF $1 A$ is one of the Ras-effectors, whereas RASGRF2 regulate the RAS GTPases cycle.

Neither RASGRF2 nor RASSF1A showed a significant association with methylation and patients' clinical characteristics. The prognostic association of RASSF $1 A$ methylation in lung cancer is a controversial issue (18-20). The lack of significance in the association of methylation with the outcome may result from the limited number of patients in our study or other factors. However, the frequent methylation of RASGRF 2 and RASSF 1A genes in lung cancer, without any correlation with clinical factors, would suggest that inactivation of these genes might be a ubiquitous mechanism stimulating tumor cell growth, rather than a means of further subdividing tumors into constituent biological or prognostic groups.

In conclusion, we demonstrated frequent inactivation of the RASGRF2 gene through aberrant methylation of the promoter in NSCLC cell lines. We also frequently found methylation of RASGRF 2 in primary NSCLC. Aberrant methylation of the $R A S G R F 2$ gene appears to be an important factor in the pathogenesis of NSCLC, a different role compared to RASSF $1 A$. Our findings of a frequent acquired tumor-related epigenetic alteration favor the candidacy of RASGRF2 as a TSG. 


\section{Acknowledgments}

This work was supported by a grant from Sasagawa Foundation Program. We thank Yusuke Suenaga, Biology Department, Faculty of Science, Chiba University, Japan, for preparing cDNA and its normalization. We greatly thank Dr Toshikazu Ushijima, Carcinogenesis Division, National Cancer Center Research Institute, Japan, for his technical advice.

\section{References}

1. Kohno T and Yokoto J: How many tumor suppressor genes are involved in human lung carcinogenesis? Carcinogenesis 20: 1403-1410, 1999.

2. Brauch H, Johnson B, Hovis J, Yano T, Gazdar A, Pettengill OS, Graziano S, Sorenson GD, Poiesz BJ and Minna J: Molecular analysis of the short arm of chromosome 3 in small cell and nonsmall cell carcinoma of the lung. N Engl J Med 317: 1109-1113, 1987.

3. Dammann R, Li C, Yoon JH, Chin PL, Bates S and Pfeifer GP: Epigenetic inactivation of a RAS association domain family protein from the lung tumour suppressor locus 3p21.3. Nat Genet 25: 315-319, 2000.

4. Pfeifer GP, Yoon JH, Liu L, Tommasi S, Wilczynski SP and Dammann R: Methylation of the RASSF1A gene in human cancers. Biol Chem 383: 907-914, 2002.

5. Burbee DG, Forgacs E, Zochbauer-Muller S, Shivakumar L, Fong K, Gao B, Randle D, Kondo M, Virmani A, Bader S, Sekido Y, Latif F, Milchgrub S, Toyooka S, Gazdar AF, Lerman MI, Zabarovsky E, White M and Minna JD: Epigenetic inactivation of RASSF1A in lung and breast cancers and malignant phenotype suppression. J Natl Cancer Inst 93: 691-699, 2001.

6. Ortiz-Vega S, Khokhlatchev A, Nedwidek M, Zhang XF, Dammann R, Pfeifer GP and Avruch J: The putative tumor suppressor RASSF1A homodimerizes and heterodimerizes with the Ras-GTP binding protein Nore1. Oncogene 21: 1381-1390, 2002.

7. Shivakumar L, Minna J, Sakamaki T, Pestell R and White MA: The RASSF1A tumor suppressor blocks cell cycle progression and inhibits cyclin D1 accumulation. Mol Cell Biol 22: 309-318, 2002.

8. Anborgh PH, Qian X, Papageorge AG, Vass WC, deClue JE and Lowy DR: Ras-specific exchange factor GRF: oligomerization through its Dbl homology domain and calciumdependent activation of Raf. Mol Cell Biol 19: 4611-4622, 1999.

9. Arozarena I, Matallanas D, Berciano MT, Sanz-Moreno V, Calvo F, Munoz MT, Egea G, Lafarga M and Crespo P: Activation of H-Ras in the endoplasmic reticulum by the RasGRF family guanine nucleotide exchange factors. Mol Cell Biol 24: 1516-1530, 2004
10. Qiu C, Yu M, Shan L and Snyderwine EG: Allelic imbalance and altered expression of genes in chromosome $2 q 11-2 q 16$ from rat mammary gland carcinomas induced by 2-amino-1-methyl6-phenylimidazo[4,5-b]pyridine. Oncogene 22: 1253-1260, 2003.

11. Hagihara A, Miyamoto K, Furuta J, Hiraoka N, Wakazono K, Seki S, Fukushima S, Tsao MS, Sugimura T and Ushijima T: Identification of $275^{\prime} \mathrm{CpG}$ islands aberrantly methylated and 13 genes silenced in human pancreatic cancers. Oncogene 23: 8705-8710, 2004.

12. Girard L, Zochbauer-Muller S, Virmani AK, Gazdar AF and Minna JD: Genome-wide allelotyping of lung cancer identifies new regions of allelic loss, differences between small cell lung cancer and non-small cell lung cancer, and loci clustering. Cancer Res 60: 4894-4906, 2000

13. Suzuki M, Toyooka S, Miyajima K, Iizasa T, Fujisawa T, Bekele NB and Gazdar AF: Alterations in the mitochondrial displacement loop in lung cancers. Clin Cancer Res 9: 5636-5641, 2003.

14. Zochbauer-Muller S, Minna JD and Gazdar AF: Aberrant DNA methylation in lung cancer: biological and clinical implications. Oncologist 7: 451-457, 2002.

15. Leblanc V, Delumeau I and Tocque B: Ras-GTPase activating protein inhibition specifically induces apoptosis of tumour cells. Oncogene 18: 4884-4889, 1999.

16. Yang JY and Widmann C: The RasGAP N-terminal fragment generated by caspase cleavage protects cells in a Ras/PI3K/Aktdependent manner that does not rely on NFkappa B activation. J Biol Chem 277: 14641-14646, 2002.

17. Suzuki M, Sunaga N, Shames DS, Toyooka S, Gazdar AF and Minna JD: RNA interference-mediated knockdown of DNA methyltransferase 1 leads to promoter demethylation and gene re-expression in human lung and breast cancer cells. Cancer Res 64: 3137-3143, 2004.

18. Tomizawa Y, Kohno T, Kondo H, Otsuka A, Nishioka M, Niki T, Yamada T, Maeshima A, Yoshimura K, Saito R, Minna JD and Yokota J: Clinicopathological significance of epigenetic inactivation of RASSF1A at 3 p 21.3 in stage I lung adenocarcinoma. Clin Cancer Res 8: 2362-2368, 2002.

19. Wang J, Lee JJ, Wang L, Liu DD, Lu C, Fan YH, Hong WK and Mao L: Value of p16INK4a and RASSF1A promoter hypermethylation in prognosis of patients with resectable non-small cell lung cancer. Clin Cancer Res 10: 6119-6125, 2004.

20. Toyooka S, Suzuki M, Maruyama R, Toyooka KO, Tsukuda K, Fukuyama Y, Iizasa T, Aoe M, Date H, Fujisawa T, Shimizu N and Gazdar AF: The relationship between aberrant methylation and survival in non-small cell lung cancers. Br J Cancer 91: 771-774, 2004. 